Interesse einfacherer Nachbehandlung ror. Die Blasendrainage schien mir durch den Verweilkatheter hinliinglich ersetzt zu sein. I)ie ideale Operation bleibt natiirlich unzweifelhaft die von der Scheide aus, welche anch nie durch diejenige ron der Blase aus erset\%t werden kann. Aber sie muss für die von unten unzugänglichen Faille im Interesse del qualroll Leidenden ihr Recht behaupten. Sie wiurde im vorliegenden Falle anch unzweifelhaft zur Heilung der Fistel gefihm haben, wenn nicht die akut auftretende Nephritis - - im lirin fanclen sich starke Eiweissmengen und viele granulöse Cylinder - das linde herbeigefuhrt hătte. Aus Furcht ror dieser, angesichts der schweren hereditaren Belastung, war mit Ausführung der Operation lange gezögert worden; die Kranke verlangte aber schliesslich dringend danach und so wurde sie, nachdem Wochen larg der Urin normale Beschatfenheit gezeigt, ausgeführt. So schwierig sie auch ist, so möchte ich sie doch für die gliicklicherweise selteneren Fialle dieser Art als rationell empfehlen.

\title{
VI. Bericht ïber weitere Erfahrungen mit einer „neuen Operation zur Heilung der Incontinentia urinae $^{66}$
}

\author{
Von \\ Dr. FRANZ T. B. FEST, \\ (in Planc Road. Sg. Nord.Amerika.)
}

Slit 1 Abbildung.

Obschon ich unter angegebenem Titel bereits im vorigen Jahre an anderem Orte ${ }^{1}$ ) diese Operation beschrieb und iiber einen Fall be-

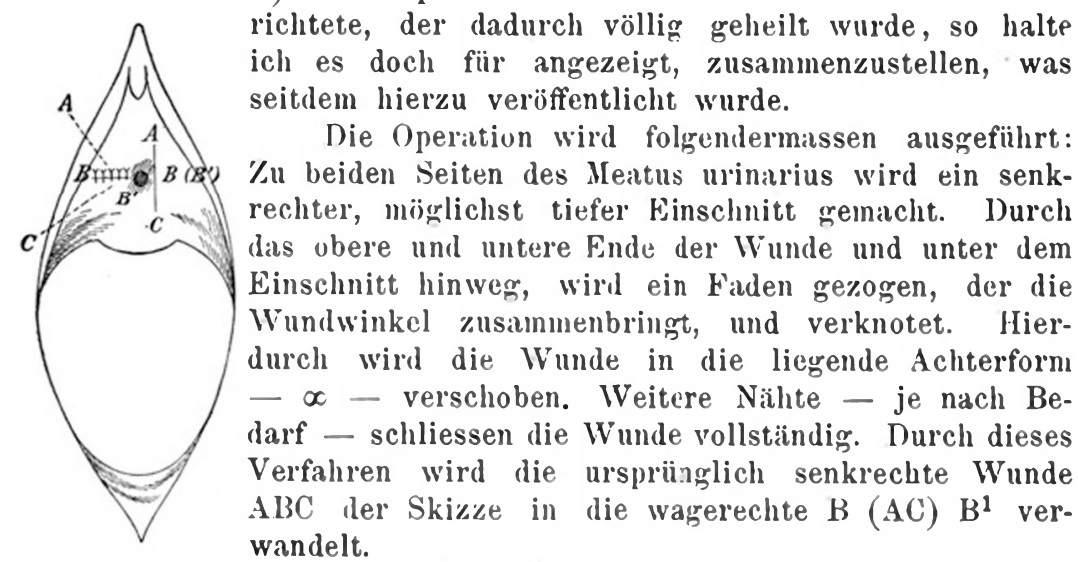

1) Franz T. B. Fest. - Eino neue Operation zur Heilung der Incontinentia urinae bei Frauen. Frauenarzt. X. Jahrg., 5. Heft, Seite 193. - $1 \varepsilon 95$. 
Diesem Nalıtverfahren liegt die Absicht zu Grunde, zu beiden Seiten der Lrethralöffnung die Wandungen \%u verdicken, das Isumen des Neatus $\approx u$ beschrinken, und hierilurch dessen Widerstandsfihigkeit zu erhöhen. Es ist in der Folge ein bedeutend grösserer Kraftaufwand nötig, um den Crin zu entlehren: denn wie Blech²) die Erfahrung machte, konnte nach der Operation die B3lase \%uerst nur in einem diinnen Strahle entleert werilen.

Mein Fall hetraf eine 24 jihlirige Frau bei der behufs Entfernung eines Steines die Crethra berleutend erweitert werden musste. Beim Husten, Niessen, bei heftigen Bewegungen, allch im Schlafe, ging der Harn unwillkiirlich ab und konnte iberhaupt nur in :iusserst geringen Mengen gehalteu werder. Das Cebel wurde durch die Operation vollständig gehoben.

Der Fall von Gustavus Blech in Detroit betraf ein 14 jiihriges Miidchen aus gesunder Fimilie mit Enuresis. Allomeinbehandlung, Massage, Elektrizitiit 11 . s. w. erwiesen sich als erfolylos. Die Patientin war sehr kriftig entwickelt fiir ihr. Alter und seit den letzten sechs Monaten regehnässig menstruiert. Dic Enuresis war für das Miidchen ein $u m$ so grösseres Uebel, da sie dienen musste und wegen ihres Leidens in keiner Stelle bleiben konnte. Die Inspektion der :iusseren Genitalien ergab nichts Abnormes.

Nach der Operation konnte diss Miidchen den Har'n zwar nur in feinem Strahle entleeren, bald aber konnte ranz normal Wasser gelassen werden.

Professor D. 'Tod Gilliam ${ }^{3}$ ) in Columbus verioffentlichte zwei Fïlle, in denen durch die gleiche Operation völlige Heilung ererzielt wurde.

Gilliam erster Fall betraf die 21 -jährige Tochter wohlhabender Eltern. Bei Vormahme der Operation zeigte sich eine Fortsetzung des Hymens mit der Trethra verwachsen. Durchtrennung desselben und Spaltung des Vestibulum zu beiden Seiten des Meatus. Vom Voll\%ur der Operation an hatte sie vollkommene Continen\%.

In zweiten Falle handelte es sich un Enuresis nocturna bei einer erwachsenen Dame; welche sich auch am 'Tilge nicht niederlegen konnte, ohne dass ihr im Schlafe Harn abying. Auch hier erwies sich meine Operation als erfolgreich.

Gilliam weist noch auf den Reflexrei\% hin, der mïglicherweise durch Bänder und Verwachsungen in der Nachbarschaft der Urethra Enuresis verursachen oder verschlimmern könne. Die Abtragung solcher Gebilde ist demnach stets angezeigrt.

Gilliam schliesst seinen Artikel mit den Worten; „Die Operation mag nicht auf alle Fiille passen, -- wunderbar falls sie es thite; - jedoch in deren Mehrzahl wird sie angezeigt sein und sich als Segen erweisen, in ihnlichem Verhälnis

2) Gustavus Blech. - A Case of Succesful 'Treatment of Fnuresis nocturna by the "Fest"-Operation. II edical-Record (New-York). - Vol. XLV'III. No. 12, page 418. - 21. Sept. 1895. -

3) D. T'od Ciflliam. - An Operation for the Cure of Incontinence of Urine in the fernale. 'T'be American Journal of(obstetrics. Vol. XXXIIl. No. 2, page 177. - Febr. 1896. 
wie die Operation zur Heilung der vesico-vaginalen Fisteln!" - Mag dieser Ausspruch auch dem Enthusiasmus des guten Erfolges entsprungen sein; jedenfalls verdient dieses einfache Verfahren den Vorzurg vor den komplizierteren Vethoden, die eine teilweise Ausschälung des Urethra, ihre 'Transplantation u. s. w. nötig machen. Das Prinzip ist das nïmliche, die Technik bedeutend harmloser und einfacher. -

\title{
Berichte.
}

\section{Ueber Asphyxia neonatorum und deren Behandlung.}

\author{
Sammelbericht \\ (bis April 1896) \\ Von \\ Dr. LUDWIG KNAPP. \\ (Prag).
}

Litteratur- Uebersicht.

1. R. Olshausen, Ueber den ersten Schrei. Berlin. Klin. Wochenschrift 1894. No. 48.

2. Runge, Die Ursache der Lungenatmung des Neugeborenen. Arch. f. Gyuaekol. Bd. XLVI p. 512 f.

3. Ahlfeld, Beitrige zur Lehre vom Uebergange der intra-uterinen Atmung zur extrauterinen. Marburg 1891.

4. Runge, Der erste Schrei und der erste Atemzug. Berlin. Klin. Wochenschrift 1895 No. 5.

5. Runge, Zur Frage nach der Ursache des ersten Atemzuges des Neugeborenen. Zeitschr. f. Gieb. I. Gyn. Bd. VI. 1881.

6. Heinricius, Zeitschr. f. Biologie Bd. XXVI N. F. VIII.

7. Engström, Skandinav. Arch. f. Physiol. Bd. II. 1891. S. 171.

8. Olshausen, 'Zur Frage des ersteu Atenzuges. Berlin. Klin. Wochenschrift 1895. No. 6 .

9. Zuntz und Strassmann, Ueber das Zustandekommen der Atmung beim Neugeborenen und die Mittel zur Wiedlerbelebung Asphrktischer. Berlin. Klin. Wochenschrift 1895̄. No. 17. Diskussion: ibid. 1895̄. No. 12. und Deutsch. med. Wochenschrift 1895. Vereinsbeilage No. 5\%.

10. Eckerlein, Zur Kenntnis des Atmungsmechanismus der Neugeborenen. Zeitschr. f. Geb. u. Gy'm. Bd. XIX. p. 1:20.

11. Rennebaum. Die Atmungsciuve des neugeboreneu Kindes. Jena 1884.

12. Knoll, Veber die Atmungsinuervation, Verhanill. d. Congr. f. innere Jeerlicin. Wiesbalen $1886^{\circ}$ p. $210 \mathrm{f}$.

13. Knoll, Beiträge zur Lehre von der Atmungsinnervation. Sitzungsbericht d. k. k. Academie d. Wissenschaften zII Wien, 1887, 11I. Abt.

14. Grossmann. Das Respirationscentrum, insbesondere des Kehlkopfes. Sond.Abdr. $n$, d. Litt.-Ber. d. k. k. Akademie d. Wissenschaften in Wien, Juli 1889.

15. Grossmann, Die Wurzelfaseru der Laryuxnerven. Wien. Klin. Wochenschrift 1889,49, u. $1890 \mathrm{I}$.

16. Heiuricius, Ueber die Bedeutung der Lungenvagi, die 'Jähigkeit des Herzens Neugeborener, über die Herzvagi bei Foeten und Nengeborenen. Zeitschr. f. Biolog. XXVI. 1889. p. 178 f. 